\title{
News
}

\section{The Coronaviruses: Past, Present, Future}

Kimball Kaufman DO, Shobhit Keswani MD,', Cristobal Cintron, MSc, DrPh, ${ }^{2}$ Hossein Akhondi, MD ${ }^{1}$

\section{Abstract}

\section{Description}

The current coronavirus pandemic is unparalleled in recent memory and fundamentally unique. It has been more than a century since humanity fought the Spanish flu pandemic. That widespread disease devastated a World War I-ravaged population when there was a relative paucity of medical knowledge. Coronavirus, on the other hand, is now befalling upon a developed world with advanced infrastructure and health systems; wherein access to medical information and technology is abundant. Yet, it has proven to be a destroyer of economies and populations without geographical partiality.

Author affiliations are listed at the end of this article.

Correspondence to: Kimball Kaufman DO Internal Medicine Residency Program MountainView Hospital Las Vegas, NV 89128 (Kimball.Kaufman@ hcahealthcare.com)

This article is meant to be a broad analysis of the virus, which is causing global disruption from both a historical and clinical perspective. We are receiving new facts and figures daily. Thus, this review is up-to-date as much as it can be and meant to serve as a general knowledge base for all involved in the fight.

\section{Keywords}

coronavirus infections; COVID-19; severe acute respiratory syndrome; SARS; SARS-CoV; SARS-CoV-2; MERS; emerging communicable diseases; infectious disease medicine; pandemics; therapeutics/drug therapy

\section{Introduction}

The coronaviruses are enveloped ribonucleic acid (RNA) viruses that are common among human and animal populations. Six known species affect humans, four of which cause common cold symptoms (strains 229E, OC43, NL63, and HKU1). The two other strains - severe acute respiratory syndrome coronavirus (SARS-CoV) and Middle East respiratory syndrome coronavirus (MERS-CoV) - are zoonotic in origin and can be fatal in humans. SARS-CoV was the causal agent of the severe acute respiratory syndrome outbreaks in 2002 and 2003 in Guangdong Province, China. MERS-CoV was the pathogen responsible for severe respiratory disease outbreaks in 2012 in the Middle East. SARS-CoV-2 (COVID-19) is the seventh known coronavirus to infect people.

All three examples are positive-strand RNA viruses, and the virion consists of a nucleocapsid core encapsulated by the three envelope glycoproteins: spike (S), membrane (M) and envelope (E). The RNA is packaged by the nucleocapsid $(N)$ protein into a helical nucleocapsid. (Figure 1) The N protein is thought to be a potential source of its pathogenicity, whereas the $\mathrm{S}$ protein is a target of the cellular immune response.

In this article, we will briefly compare the three outbreaks in terms of historical background, epidemiology, virology, clinical picture and lasting impact. Regarding COVID-19, we have synthesized data from the most significant known studies to provide a summary of clinical signs and symptoms, imaging findings, case fatality, current treatment options and lastly, specific steps that healthcare providers can take to prevent, manage and prepare for the future of this evolving pandemic.

\section{Key Epidemiologic Terms}

A common outbreak refers to an exposure of

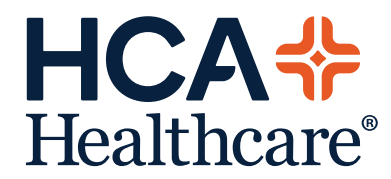

www.hcahealthcarejournal.com

(c) 2020 HCA Physician Services, Inc. d/b/a Emerald Medical Education

\author{
HCA Healthcare \\ Journal of Medicine
}




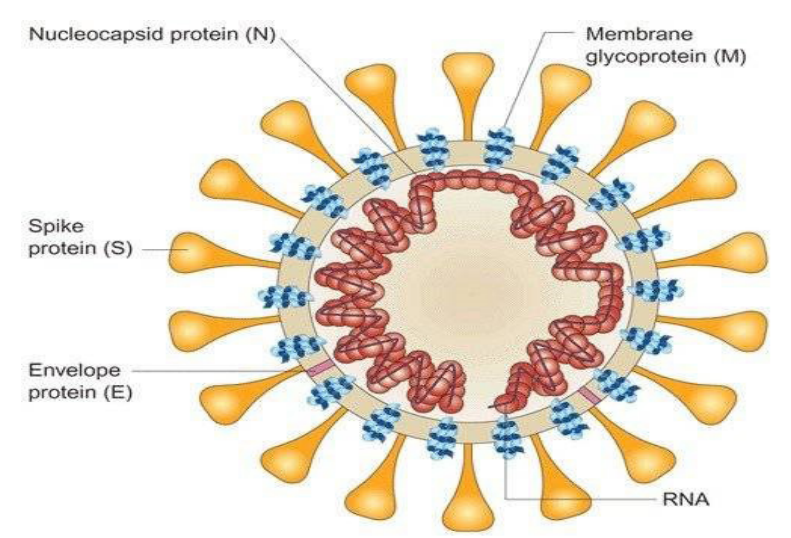

Figure 1. SARS-CoV Nucleocapsid and Glycoproteins Credit: Assay Genie. Published with permission.

a susceptible population to a common source of a pathogen at the same time, such as at a restaurant or picnic. The term endemic reflects the occurrence of a disease at a relatively constant level in a given setting.

An epidemic is the occurrence of cases of illness, specific health-related behavior or other health-related events clearly above average expectancy in a community or region. The number of cases indicating the presence of an epidemic varies according to the agent, size and type of the population exposed, previous experiences, lack of exposure to the disease, and time and place of occurrence. Epidemicity is thus a relative term. Two cases of a disease long absent from a population or the first invasion by a disease not previously recognized, associated in time and place, may be sufficient to be considered an epidemic. ${ }^{2}$

A pandemic refers to an epidemic occurring over an extensive area and usually affecting a large portion of the population. ${ }^{3}$ Key pandemics from the past include the 1918 Spanish flu (50100 million deaths), 1957 Asian flu (1.1 million deaths), 1968 Hong Kong flu (1 million deaths), 1980s AIDS pandemic (25 million fatalities) and the 2009 Swine flu (100,000 to 400,000 deaths). The WHO pronounced COVID-19 a pandemic on March 12, 2020. (Table 1)

Table 1. Comparison of Coronaviruses

\begin{tabular}{|c|c|c|c|c|}
\hline Disease & Flu & SARS & MERS & COVID-19 \\
\hline Pathogen & Influenza virus & SARS-CoV & MERS-CoV & SARS-CoV-2 \\
\hline Country of origin & Worldwide & China & Saudi Arabia & Wuhan, China \\
\hline First outbreak & 1918 & 2003 & 2012 & 2019 \\
\hline Type of the virus & RNA & RNA & RNA & RNA \\
\hline Pathogen origin (zoonotic) & Avian, Swine, etc. & Bats, Civet & Bats, Camel & Bats \\
\hline Incubation in days & $1-4$ & $2-7$ & 6 & $4-14$ \\
\hline Community attack rate & $10-20 \%$ & $10-60 \%$ & $4-13 \%$ & $30-40 \%$ \\
\hline Hospitalization rate & $2 \%$ & Most cases & Most cases & $\sim 19 \%$ \\
\hline Fatality rate & $0.05-0.01 \%$ & $9.6-11 \%$ & $34.40 \%$ & $-3.40 \%$ \\
\hline Total deaths (WHO) & $\begin{array}{l}12,000-61,000 \\
\text { annually }\end{array}$ & 916 & 858 & Ongoing \\
\hline
\end{tabular}

Created using BioRender.com Credit: Keswani S. Department of Internal Medicine, MountainView Hospital. 


\section{SARS Epidemic, 2003}

The SARS virus originated in the province of Guangdong in southern China in November 2002, where it initially was thought to have arisen from bat-to-civet transmission. ${ }^{4}$ Civets are a food source in China, and although they have long been suspected of playing a role in SARS transmission, SARS experts have stopped short of saying the animals are the natural reservoir of the virus or its conveyor to humans. This strain was discovered by Italian physician Dr. Carlo Urbani in February 2003. Later that year, he would succumb to the disease. The CDC has noted that viruses very similar to SARS-CoV have been found in civets and that some civet handlers had evidence of infection with SARS-CoV or a very similar illness.

During the outbreak, which lasted just six months, more than 8000 patients were infected, and 900 died throughout 29 countries. This resulted in a mortality rate of approximately $9 \%$. More than half of the cases and roughly one-third of the deaths occurred in mainland China. There were only eight laboratory-confirmed cases in the US. No cases of SARS have been reported anywhere in the world since 2004. . $^{6}$ (Figure 2)

Like COVID-19, the mortality rate was higher in the elderly, approaching $50 \%$ for this subset of patients. Notably, more than one-third of the initial cases occurred in food handlers and health care workers. The SARS outbreak was an epidemic based on population reports.

\section{MERS Outbreak, 2012}

Middle East respiratory syndrome-coronavirus (MERS-CoV) is also a zoonotic coronavirus that has infected humans time and again via direct or indirect contact with dromedary camels in the Arabian Peninsula. The evidence available to date suggests that the virus was spread from bats to camels sometime in the mid-1990s in South Korea. The virus then appeared to have spread from camels to humans in the early 2010s. ${ }^{7}$ The original bat host species is unknown, and MERS-CoV has a different genetic makeup from SARS and COVID-19. It is an enveloped, positive-sense, ss-RNA virus that enters cells by binding to the Dipeptidyl peptidase-4 (DPP4) receptor, which is also known as CD-26. In humans, the virus has a predilec-

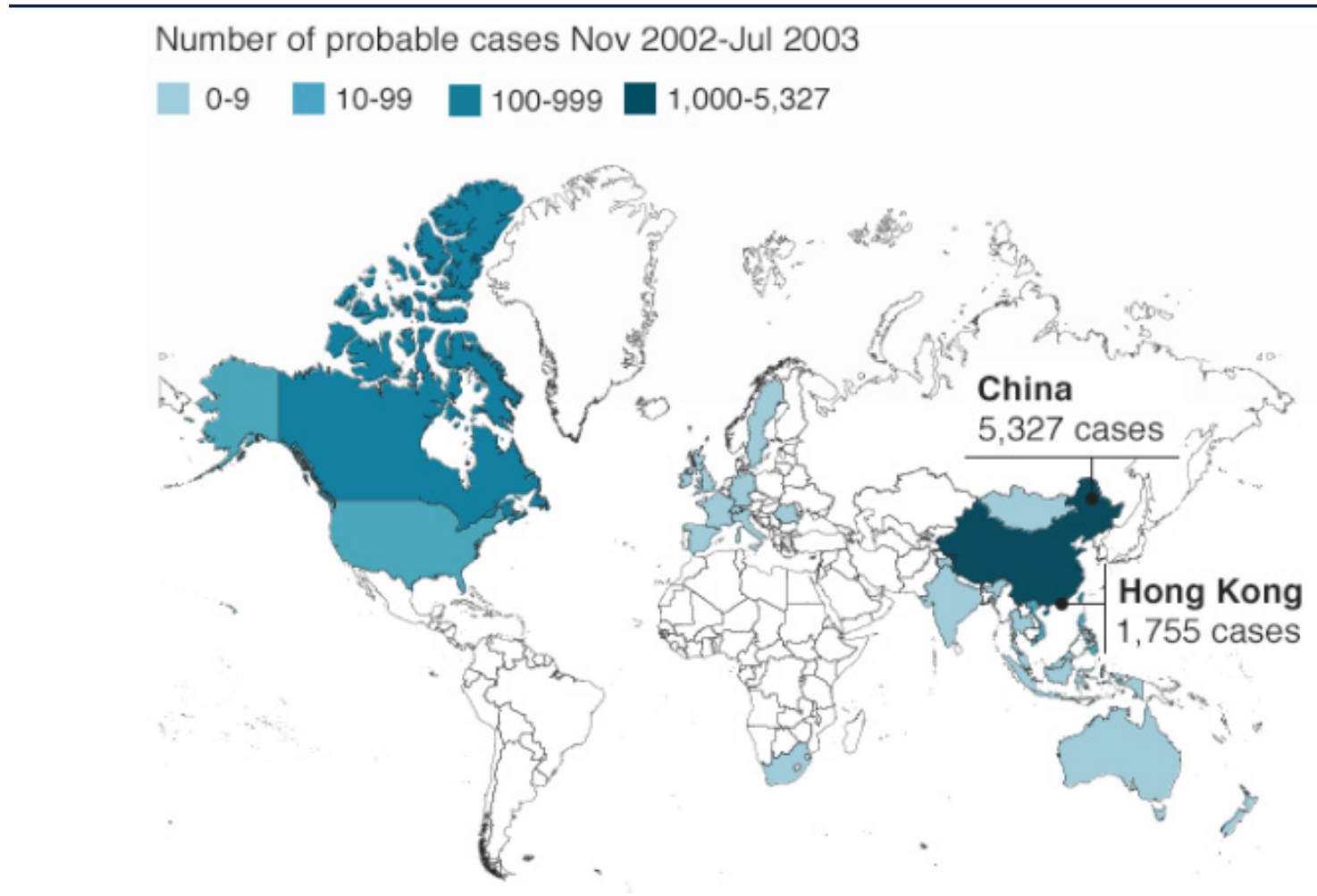

Figure 2. SARS Epidemic Source: WHO 


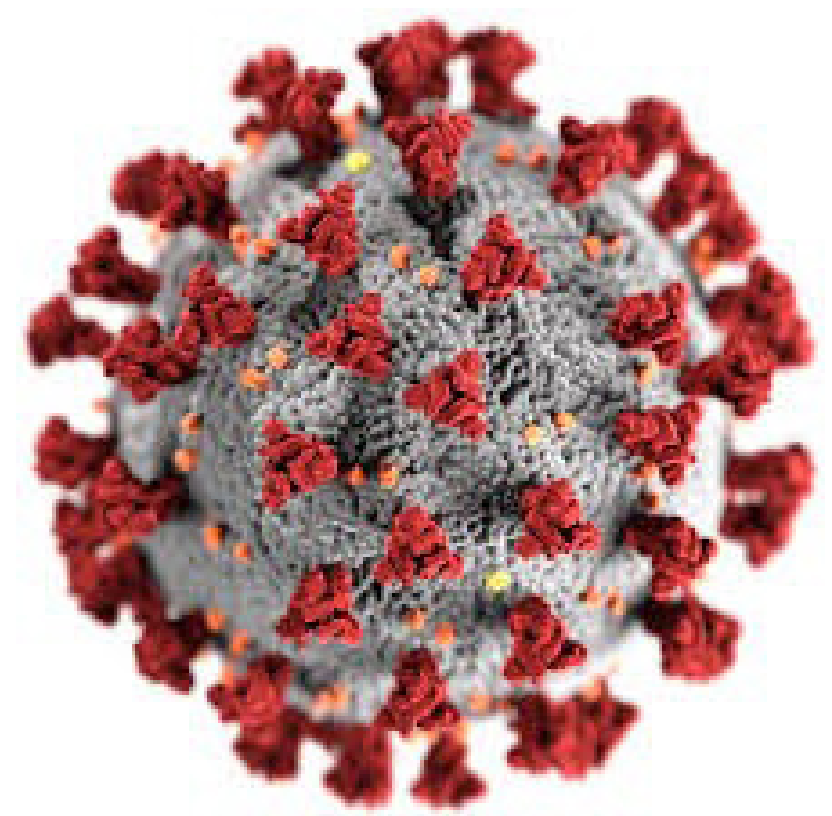

Figure 3. SARS-COVID Solar Corona Appearance Credit: Helmholtz-Zentrum Berlin

tion for non-ciliated bronchial epithelial cells. These makeup approximately $20 \%$ of respiratory epithelial cells, so large numbers of virions are required to cause a severe infection. This mechanism is unusual in that most respiratory viruses target ciliated cells. The amino acid sequence of DPP4 is found in both bronchial epithelium and the kidneys, which explains why the virus affects the lung and causes kidney insufficiency. The virus also appears to have the ability to elude innate immune responses and subdue interferon production.

Between 2012 and 2017, over 2000 laboratory-confirmed cases of MERS-CoV infection were reported. Over $80 \%$ of the infections occurred in Saudi Arabia. The largest focus outside of the Middle East occurred in Korea with nearly 200 cases. Only two patients in the US ever tested positive for MERS. ${ }^{7}$ The mortality rate was initially reported at near an astonishing $100 \%$ and then decreased to $35 \%$.

No sustained human-to-human transmission has occurred anywhere in the world. However, limited, non-sustained, human-to-human transmission in health-care facilities was a prominent feature of this virus. The WHO never classified this as a health emergency. ${ }^{6}$

\section{SARS-CoV-2 (COVID-19)}

Pandemic, 2019-2020

SARS-CoV-2 (COVID-19) is the seventh known coronavirus to infect humans. Like the SARS-related coronavirus strain implicated in the 2003 SARS outbreak, COVID-19 is a member of the subgenus Sarbecovirus. Its RNA sequence is approximately 30,000 bases in length. Among the human coronaviruses, SARS-CoV has exhibited the most genome similarity to COVID-19 at around $80 \%$. MERS, on the other hand, shares only about $50 \%$ sequence similarity with COVID-19. Notably, COVID-19 is nearly identical to a coronavirus found in bats.

This new strain is novel for its integration of a polybasic cleavage site, a feature known to increase pathogenicity and transmissibility. Electron micrographs of virion particles reveal a spherical shape with pleomorphism. The particles have distinctive spikes and give the virions a solar corona appearance. (Figure 3) The diameter varies from about 60 to $140 \mathrm{~nm}$. Like other coronaviruses, the spike protein is responsible for allowing the virus to attach to the membrane of a host cell. Protein modeling experiments focusing on the spike protein reveal that COVID-19 has an affinity to bind angiotensin-converting enzyme 2 (ACE2) receptors, which are abundant in the lungs. Studies have 
also shown that COVID-19 has a higher affinity to human ACE2 receptors than the 2003 SARS strain. COVID-19 produces at least three virulence factors that promote the dissemination of new virions from host cells and inhibit the immune response. ${ }^{8}$

The nidus of the outbreak appears to have been the Wuhan seafood market in China. It is unclear which animal transferred the virus to humans-bat, snake and pangolin have all been suggested as potential carriers. The market in Wuhan is not an unfamiliar environment in China. Across mainland China, hundreds of similar markets offer a wide range of exotic animals for a variety of purposes. The danger of an outbreak comes when many exotic animals from different environments are kept closely together, establishing a setting for cross-species transmission.

As of April 2020, worldwide cases have surpassed 3 million. A large percentage of the initial cases were residents of Wuhan or those who had contact with residents of Wuhan. In China, an estimated 3000 healthcare workers have been infected. ${ }^{9}$ The median age of the patients was 47 years, and only $1 \%$ of the patients were younger than 15 years of age..$^{10}$ There are nearly 1 million cases in the U.S., with more than 60,000 deaths reported. This number is expected to increase in the coming weeks. Italy, Spain and the United Kingdom have been heavily impacted as well. (Figure 4)

\section{Clinical Signs and Symptoms of COVID-19}

Fever is the most common symptom and is present on admission in approximately $40 \%$ of patients. However, nearly $90 \%$ develop a fever at some point during hospitalization. The absence of fever occurs more frequently in COVID-19 than in SARS-CoV and MERS-CoV. As a result, afebrile patients may be missed if screening solely focuses on the detection of temperature. The second most common symptom is a non-productive dry cough, which occurs in about two-thirds of patients. Less common symptoms include fatigue, sore throat, nausea, vomiting and diarrhea. Epidemiologic data from China have shown that approximately one-quarter of patients have at least one pre-existing medical condition. (Figure 5)

\section{Laboratory and Radiological Findings}

On admission, lymphocytopenia is the most common lab abnormality, occurring in greater than $80 \%$ of patients. Patients with severe disease tend to have more significant lymphocytopenia and leukopenia than those with milder disease forms. Other lab features include thrombocytopenia, transaminitis and elevated inflammatory markers such as C-reactive protein, creatinine kinase and D-dimer."

Testing for COVID-19 is evolving with time. The first test to be released, and still the most widely used, is a nucleic acid amplification test. More specifically, it is a reverse transcrip-

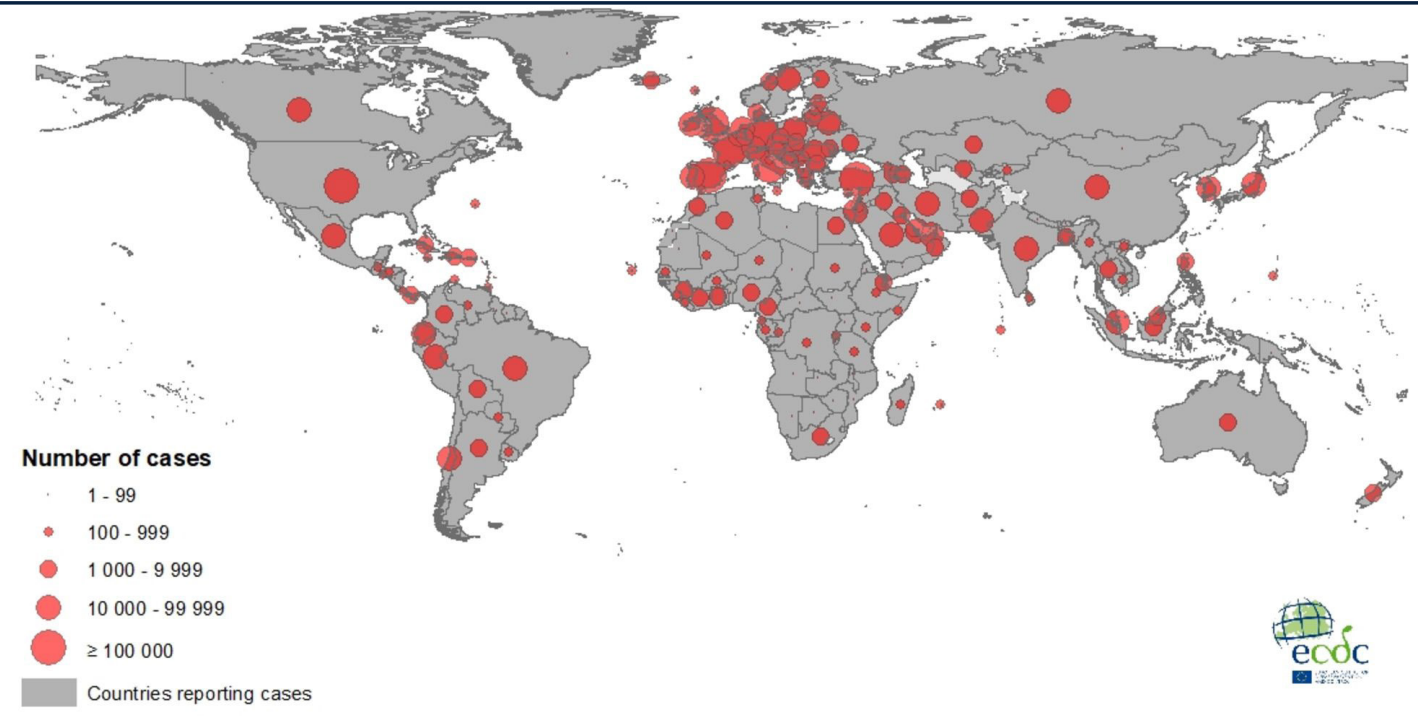

Figure 4. COVID-19 Pandemic 


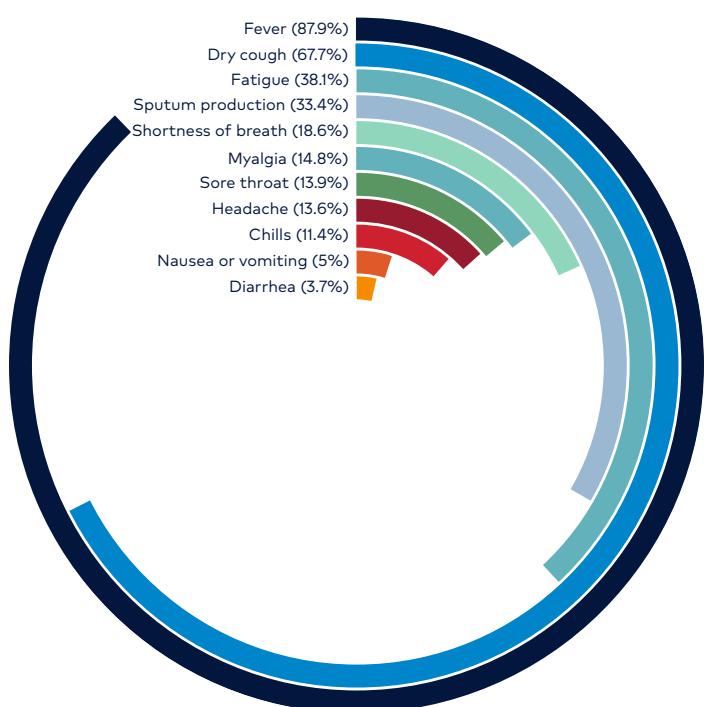

Figure 5. COVID-19 Percentage Frequency of Symptoms Credit: WHO

tase-polymerase chain reaction (RT-PCR) test, which tests for the presence of viral RNA. More recently, an lgM test has been released. However, the CDC warns that antibody testing results should not be used as the sole test to diagnose, to rule out or to inform the infection status of COVID-19. This is mainly because of the potential cross-reactivity of the antibody (poor specificity) with non- COVID-19 coronavirus strains (ex. common cold coronaviruses). The Infectious Diseases Society of America (IDSA) suggests that these antibody tests would be better used for public health surveillance and vaccine development instead. Conversely, for a patient who is symptomatic, a nucleic acid amplification test such as RT-PCR will have a high positive predictive value because of their high specificity. ${ }^{12}$

Chest computed tomography (CT) is one of the primary modalities in the diagnostic algorithm for patients with suspected COVID-19 infection. Given the limited testing capability, the National Health Commission of the People's Republic of China has promoted diagnosis based on clinical and chest CT findings alone. In a study of over 900 Chinese patients diagnosed with COVID-19, more than $86 \%$ of scans were abnormal. The most common patterns on chest CT are ground-glass opacities (56\%) and bilateral patchy infiltrates (52\%). Radiographic or CT abnormality was absent in only $3 \%$ of patients with severe disease and approximately $20 \%$ of patients with mild forms. (Figure 6)

The pattern of ground-glass and consolidative pulmonary opacities, often with a bilateral and peripheral lung distribution, seems to be a radiologic hallmark of COVID-19 infection. (Figure 7) This pattern of disease is somewhat similar to that described in earlier coronavirus outbreaks such as SARS and MERS. Other common radiologic findings with pulmonary disease such as lymphadenopathy, pleural ef-

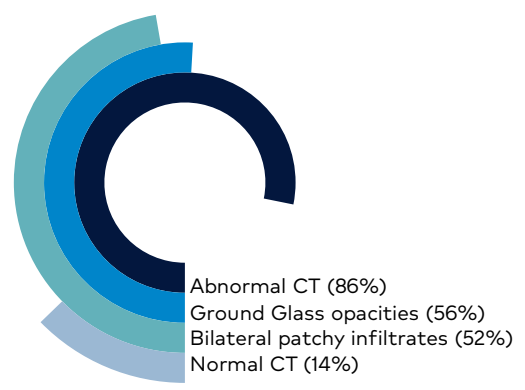

Figure 6. CT Scan Findings in Confirmed Cases of COVID-19

Credit: Keswani S. Department of Internal Medicine. MountainView Hospital. 

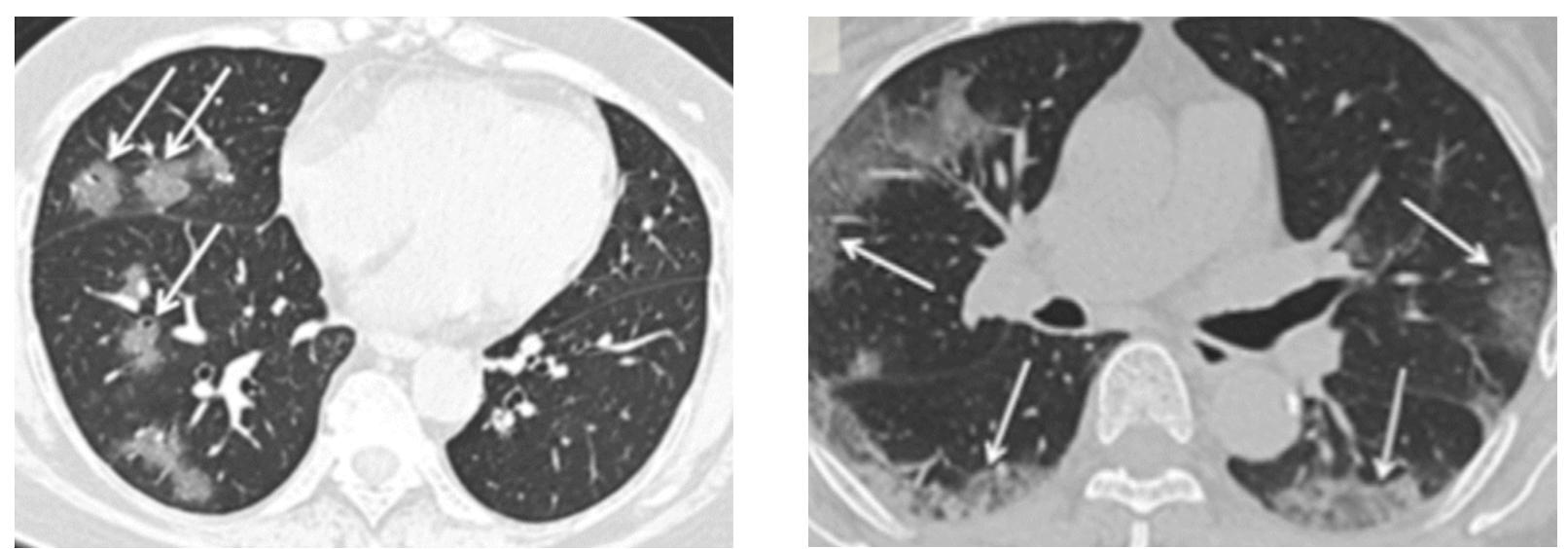

Figure 7. CT Scans with Typical Findings in COVID-19 Patients

Left: An axial CT image shows ground-glass opacities with a rounded morphology in the right middle and lower lobes.

Right: An axial CT image shows bilateral ground-glass and consolidative opacities with a peripheral distribution.

Credit: Department of Radiology, Icahn School of Medicine at Mount Sinai, New York. Reproduced with permission.

fusions, pulmonary nodules and lung cavitation are usually absent.

In a US study of 121 patients with confirmed COVID-19 infection, after 0-2 days of onset of symptoms, $56 \%$ had a normal CT with a complete absence of ground-glass opacities or consolidation. This was in contrast with less than $10 \%$ of patients with normal CT, who presented later in the disease course. ${ }^{13}$ Chest CT, therefore, has limited sensitivity and is time-dependent.

\section{Case Fatality}

Despite the number of deaths associated with COVID-19, it appears to have a lower case fatality rate than SARS-CoV and MERS-CoV. Case fatality varies by age, with older age groups having higher rates than younger ones, as represented by Figure 8. Most recent estimates by WHO reports that $3.4 \%$ out of all the people reported infected with COVID-19 have died. We expect this number to drop as more people are tested and milder cases surface. Of note, case fatality can also vary widely by comorbidity and baseline health status. Critically ill hospitalized patients with shock, respiratory failure and multi-organ failure have the highest fatality rate.

\section{Treatment}

Supportive care with supplemental oxygen is the mainstay of treatment for severe cases. For hypoxic patients, humidified oxygen via nasal cannula 1-8 liters per minute (LPM) should be employed to keep oxygen saturation greater than $92 \%$. If the patient remains hypoxic, non-humidified oxygen should be administered via Venturi mask at 9 LPM with FiO2 of 0.28 and up-titration as needed to keep oxygen saturation greater than $92 \%$. If $\mathrm{FiO} 2$ requirements exceed 0.60, intubation with video laryngoscope by the most experienced medical staff present should be employed. ${ }^{14}$ If a patient is in ARDS, the tidal volume should be set to $6 \mathrm{mg} /$ $\mathrm{kg}$ of ideal body weight, with a respiratory rate of 14-20, and positive end-expiratory pressure (PEEP) of at least $10 \mathrm{~cm} \mathrm{H2O}$. Low PEEP is not effective in COVID-19 (based on anecdotal reports from field hospitals in (taly). Sedation should be implemented if intubated, and prone positions are likely beneficial based on prior evidence in patients with ARDS. Inhalers should be used in lieu of nebulizers to reduce aerosolization of the virus.

No antiviral therapy has been proven to be a definitive cure or treatment for COVID-19 in humans. Multiple randomized controlled trials are ongoing. The vast majority of patients will do fine without any therapy, so in most cases, there's no need for antiviral treatment.

Remdesivir is being investigated in both randomized open-label trials, as well as a dou- 


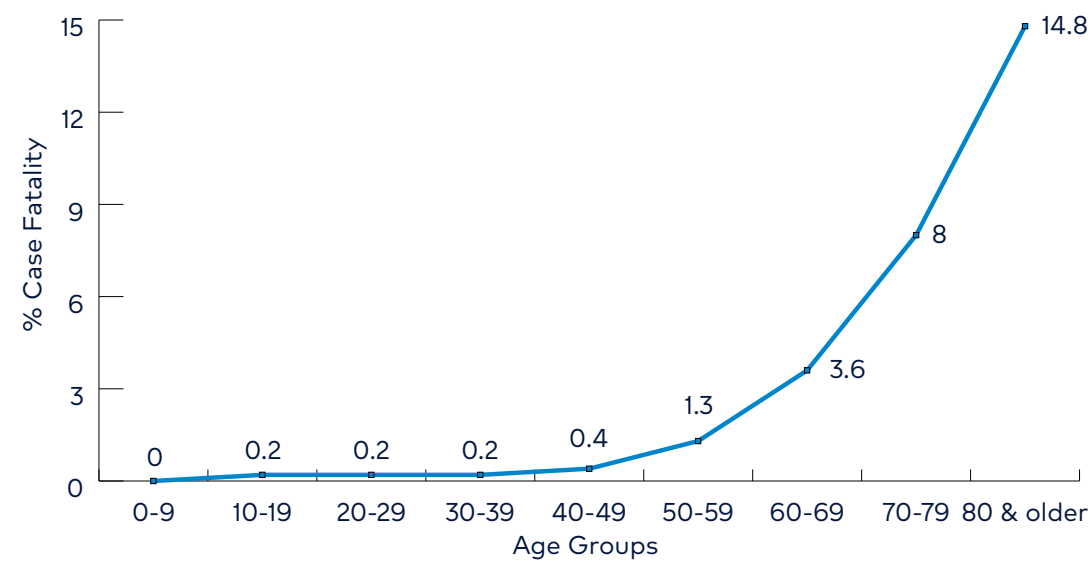

COVID-19 Case Fatality in Percent by Comorbidity

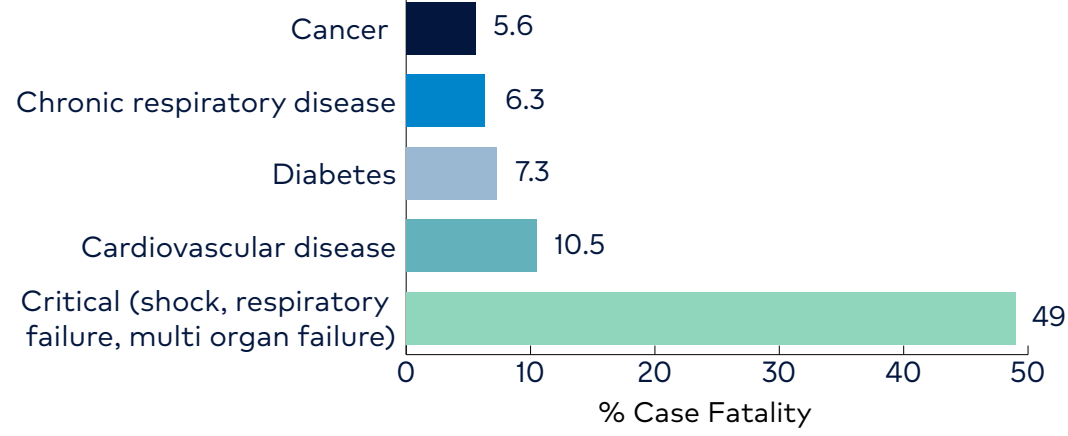

Figure 8. COVID-19 Case Fatality Source: CDC (top) and WHO (bottom)

ble-blind, randomized controlled trial involving up to 50 sites globally. ${ }^{15}$ Although not commercially available, Remdesivir has been used under the umbrella of "compassionate use".

Lopinavir/ritonavir is a combination pill for human immunodeficiency syndrome. It is more widely available than remdesivir and has an established toxicity profile. Previously, there has been evidence to show the in-vitro activity of this drug against SARS CoV. ${ }^{16}$ However, an open-label, randomized controlled trial comprising of 199 adults with confirmed COVID-19 infection showed that no benefit was observed beyond standard care when using lopanavir/ ritonavir. ${ }^{17}$ Hence its use is not recommended at this time.

The now widely popularized non-antiviral medication hydroxychloroquine is being used in many facilities. Hydroxychloroquine has shown in-vitro activity against COVID-19 and has much broader availability in the United States. ${ }^{18}$ These antimalarial drugs work via multiple mechanisms, such as interfering with the ACE2 receptor, a potentially important target. They are also involved with impairment acidification of endosomes, which can stop intra-cellular viral trafficking. However, it should be noted that these drugs have significant adverse effects, including QT prolongation and ventricular arrhythmias, which may be fatal in critically ill patients. As more evidence is released, the use of this medication is becoming controversial. The first large multicenter randomized controlled trial on hydroxychloroquine consisting of 150 patients that compared it to the standard of care alone, revealed that there was no difference in the rate of improvement of symptoms and viral clearance at 28 days. ${ }^{19}$ This study has yet to be peer-reviewed.

The role of corticosteroids has also been evaluated. Clinical evidence thus far does not support its use. Data from prior studies on SARS and MERS show that the clinical outcomes and complications outweigh any benefit that they may provide. Corticosteroids reduce inflam- 
mation but also impair the immune response. When SARS and MERS patients were given steroids, there was a delayed clearance of viral RNA. Complications in SARS patients receiving corticosteroids included psychosis, diabetes and avascular necrosis. ${ }^{20}$

Given the known role of type 2 angiotensin-converting enzyme (ACE2) in the pathogenesis of COVID-19, there has been increasing concern about whether people who are on ACE inhibitors or angiotensin receptor blockers (ARB) medications are at increased risk or have a higher susceptibility to COVID-19 infection. ACE inhibitors are common anti-hypertensive agents prescribed widely across all populations. An ACE inhibitor will inhibit angiotensin-converting enzyme 1 (ACE1), which will upregulate the angiotensin-converting enzyme 2 (ACE2). Hence people who are taking ACE inhibitors will have higher levels of ACE2.

No conclusive data are yet available regarding a relationship between these agents and poor outcomes. While ACE2 levels may be increased in such patients, the evidence is limited for an increase in pulmonary ACE2 levels. Moreover, ACE inhibitors have found to be protective in limited lung injury models in mice. In one such study, SARS-CoV downregulated ACE2, worsening lung injury, and treatment with ARBs improved outcomes. Also, patients on these drugs tend to be older with other comorbidities that may explain their increased mortality. Professional organizations such as the American College of Cardiology, European Society of Cardiology, and the American Heart Association have recommended that patients continue their anti-hypertensive treatment with ACE inhibitors or ARBs as prescribed. ${ }^{21}$

Interleukin 6 (IL-6) activates T cells and macrophages, and has been approved in the past for cytokine activation syndrome and its complications..$^{22}$ IL-6 levels are possibly correlated with severe COVID-19 based on bronchoalveolar lavage of these patients, which show lymphocytosis (despite peripheral leukopenia). IL-6 inhibition, therefore, might be helpful. There are anecdotal reports of the successful use of tocilizumab in Italy.

A phase 1 clinical trial for an investigational vaccine has been started by the National Institute of Health for COVID-19. Current estimates for approval and availability range from 12-18 months.

Of all the above therapies discussed, at this point, remdesivir seems to be the most promising for a multitude of reasons. First, remdesivir has shown good in vitro activity against many human and zoonotic coronaviruses, including the SARS-CoV and bat coronaviruses. Therefore it can act as a broad-spectrum antiviral agent against coronaviruses. Second, remdesivir resistance has been studied, and findings indicate a high genetic barrier for resistance to remdesivir to develop. Moreover, remdesivir has also been studied in murine models with SARSCoV, among others, showing that early therapeutic dosing reduced viral load and improved pulmonary function. Intravenous remdesivir therapy was shown to have a compelling safety profile in two case reports. Two randomized clinical trials of parenteral remdesivir therapy for COVID-19 are currently underway in China. For these reasons, increased use of remdesivir is highly anticipated. ${ }^{23}$

\section{Future}

We should expect to see an increase in the number of cases and high death tolls until a significant amount of the population transitions from susceptible to immune. Healthcare providers must pay attention to epidemiological terms and methods that will be necessary to learn and use for this new challenge. The understanding of risk factors, paths of transmission, epidemiological surveillance efforts and the use of information for intelligent allocation of resources is necessary.

What practical measures can health care workers employ to protect themselves?

1. Making smart use of personal protective equipment (PPE), conserve when possible.

2. Practice smart and effective social distancing, even with colleagues, within the healthcare setting.

3. Limit unnecessary length and frequency of interaction between health care personnel and patients.

4. Encourage patient face mask usage, especially for confirmed COVID-19 positive patients.

5. Defer elective or non-urgent care. 


\section{Conflicts of Interest}

The authors declare they have no conflicts of interest.

Drs. Kimball Kaufman, Shobhit Keswani and Hossein Akhondi are employees of Mountain View Hospital, a hospital affiliated with the journal's publisher.

Dr. Cristobal Cintron is an employee of HCA Healthcare North Florida Division GME, an organization affliated with the journal's publisher.

This research was supported (in whole or in part) by HCA Healthcare and/or an HCA Healthcare affiliated entity. The views expressed in this publication represent those of the author(s) and do not necessarily represent the official views of HCA Healthcare or any of its affiliated entities.

\section{Author Affiliations}

1. MountainView Hospital, Internal Medicine Residency, Sunrise Health GME Consortium, Las Vegas, NV

2. University of Central Florida/HCA Healthcare North Florida Division GME

\section{References}

1. McBride R, Fielding BC. The role of severe acute respiratory syndrome (SARS)-coronavirus accessory proteins in virus pathogenesis. Viruses. 2012;4(11):2902-2923. Published 2012 Nov 7. https://doi.org/10.3390/v4112902

2. Last JM, ed. A Dictionary of Epidemiology. Third Ed. New York: Oxford University Press; 1995.

3. Kelsey JL. Methods in Observational Epidemiology. New York: Oxford University Press; 1996.

4. Drosten C, Günther S, Preiser W, et al. Identification of a novel coronavirus in patients with severe acute respiratory syndrome. $N$ Engl J Med. 2003;348(20):1967-1976. https://doi.org/10.1056/ NEJMoa030747

5. World Health Organization. Agenda item 14.16: Severe acute respiratory syndrome (SARS). Resolution to the WHO World Health Assembly. 2003 May. https://www.who.int/csr/sars/en/ ea56r29.pdf

6. Severe Acute Respiratory Syndrome (SARS) Epidemiology Working Group. Consensus document on the epidemiology of severe acute respiratory syndrome (SARS). World Health Organization. https://www.who.int/csr/sars/en/ WHOconsensus.pdf
7. WHO MERS-CoV Global Summary and Assessment of Risk. World Health Organization; 2017. https://www.who.int/emergencies/mers-cov/ risk-assessment-july-2017.pdf

8. Walls AC, Park YJ, Tortorici MA, Wall A, McGuire AT, Veesler D. Structure, function, and antigenicity of the SARS-CoV-2 spike glycoprotein. Cell. 2020;181(2):281-292.e6. https://doi.org/10.1016/j. cell.2020.02.058

9. Adams JG, Walls RM. Supporting the Health Care Workforce During the COVID-19 Global Epidemic. JAMA. 2020;323(15):1439-1440. https:// doi.org/10.1001/jama.2020.3972

10. Zhu N, Zhang D, Wang W, et al. A novel coronavirus from patients with pneumonia in China, 2019. N Engl J Med. 2020;382(8):727-733. https:// doi.org/10.1056/NEJMoa2001017

11. Guan WJ, Ni ZY, Hu Y, et al. Clinical characteristics of coronavirus disease 2019 in China [published online ahead of print, 2020 Feb 28]. N Engl J Med. 2020;NEJMoa2002032. https://doi. org/10.1056/NEJMoa2002032

12. Infectious Diseases Society of America: IDSA COVID-19 Antibody Testing Primer, April 2020. https://www.idsociety.org/globalassets/idsa/ public-health/covid-19/idsa-covid-19-antibody-testing-primer.pdf

13. Bernheim A, Mei X, Huang M, et al. Chest CT findings in coronavirus disease-19 (COVID-19): Relationship to duration of infection [published online ahead of print, 2020 Feb 20]. Radiology. 2020;200463. https://doi.org/10.1148/radiol.2020200463

14. Alhazzani W, Møller MH, Arabi YM, et al. Surviving sepsis campaign: Guidelines on the management of critically ill adults with coronavirus disease 2019 (COVID-19) [published online ahead of print, 2020 Mar 27]. Crit Care Med. 2020;10.1097/CCM.0000000000004363. https:// doi.org/10.1097/CCM.0000000000004363

15. Adaptive COVID-19 treatment trial (ACTT). ClinicalTrials.gov identifier: NCT04280705 https:// clinicaltrials.gov/ct2/show/NCT04280705. Updated April 20, 2020.

16. Chu CM, Cheng VC, Hung IF, et al. Role of lopinavir/ritonavir in the treatment of SARS: initial virological and clinical findings. Thorax. 2004;59(3):252-256. https://doi.org/10.1136/thorax.2003.012658

17. Cao B, Wang Y, Wen D, et al. A trial of lopinavir-ritonavir in adults hospitalized with severe covid-19 [published online ahead of print, 2020 Mar 18]. N Engl J Med. 2020;NEJMoa2001282. https://doi.org/10.1056/NEJMoa2001282

18. Wang $\mathrm{M}$, et al. Remdesivir and chloroquine effectively inhibit the recently emerged novel coronavirus (2019-nCoV) in vitro. Cell Res. 2020;30(3):269-271. https://doi.org/10.1038/ s41422-020-0282-0

19. Tang W, et al. Hydroxychloroquine in patients 
with COVID-19: an open-label, randomized, controlled trial. medRxiv. 2020.04.10.20060558. https://doi.org/10.1101/2020.04.10.20060558

20. Russell CD, Millar JE, Baillie JK. Clinical evidence does not support corticosteroid treatment for 2019-nCoV lung injury. Lancet. 2020;395(10223):473-475. https://doi.org/10.1016/ S0140-6736(20)30317-2

21. Patel AB, Verma A. COVID-19 and angiotensin-converting enzyme inhibitors and angiotensin receptor blockers: What is the evidence? [published online ahead of print, 2020 Mar 24]. JAMA. 2020. https://doi.org/10.1001/ jama.2020.4812

22. Brudno JN, Kochenderfer JN. Recent advances in CAR T-cell toxicity: Mechanisms, manifestations and management. Blood Rev. 2019;34:4555. https://doi.org/10.1016/j.blre.2018.11.002

23. Ko W, et al. Arguments in Favour of Remdesivir for Treating SARS-CoV-2 Infections. J Antimicrob Agents. 2020;55(4):105933. https://doi. org/10.1016/j.ijantimicag.2020.105933 